\title{
Image Fusion Algorithm Using the Multiresolution Directional-Oriented Hermite Transform
}

\author{
Sonia Cruz-Techica and Boris Escalante-Ramirez \\ Facultad de Ingeniería, Universidad Nacional Autónoma de México, \\ Edif. Bernardo Quintana, Circuito exterior, Cd. Universitaria, México, D.F. 04510 \\ cruz_s@uxmcc2.iimas.unam.mx, boris@servidor.unam.mx
}

\begin{abstract}
The Hermite transform is introduced as an image representation model for multiresolution image fusion with noise reduction. Image fusion is achieved by combining the steered Hermite coefficients of the source images, then the coefficients are combined with a decision rule based on the linear algebra through a measurement of the linear dependence. The proposed algorithm has been tested on both multi-focus and multi-modal image sets producing results that exceed results achieved with other methods such as wavelets, curvelets 11, and contourlets [2] proving that our scheme best characterized important structures of the images at the same time that the noise was reduced.
\end{abstract}

Keywords: image fusion, Hermite transform, multiresolution, linear dependence.

\section{Introduction}

Image fusion can be defined as the process of combining information from different sources, in order to detect strong salient features in the input images and fuse these details into the fused image. In general, image fusion proposes the integration of disparate and complementary data to improve the information that appears on images as well as increased reliability and performance, which results in greater accuracy of data.

Fusion techniques can be divided into spatial domain and transform domain techniques [7]. In the first case, the input images are fused into spatial domain, the fusion process deals with the original pixel values. In contrast, in the transform domain techniques it is possible to use a framework where the salient features of the images are clearer than in the spatial domain.

In the literature several methods of pixel-level fusion have been reported which use a transformation to perform data fusion, some of these transformations are: the discrete wavelet transform (DWT) [1], the contourlet transform (CW) [15], the curvelet transform (CUW) [8], and the Hermite transform (HT) [4], 5].

The wavelet transform has been the most used technique for the fusion process but it is the technique with more problems in the analysis of signals from two or

J.-F. Martínez-Trinidad et al. (Eds.): MCPR 2011, LNCS 6718, pp. 202-210, 2011.

(C) Springer-Verlag Berlin Heidelberg 2011 
more dimensions; an example of this is the points of discontinuity that sometimes are undetected; another drawback is its limitation to capture directional information. The contourlet and the curvelet transforms have shown better results than the wavelet transform due to multi-directional analysis, but they require an extensive orientation search at each level of the decomposition. Because of this, the Hermite transform provides significant advantages to the process of image fusion: first this model of representation includes some properties of human visual system such as the local orientation analysis and the Gaussian derivative model of primary vision [16] and it also has the additional advantage of reducing noise without introducing artifacts.

In this work, we take it as a prerequisite that the source images must be registered so that the corresponding pixels are aligned. The proposed scheme fuses images on a pixel-level using a multiresolution directional-oriented Hermite transform of the source images by means of a decision map. This map is based on a linear dependence test of the rotated Hermite coefficients.

The rest of the paper is organized as follows: Section 2 presents the basic concepts of Hermite Transform. Section 3 describes the proposed image fusion algorithm. Section 4 focuses on experiments, evaluation criteria and analysis of results. Finally conclusions are introduced in section 5 .

\section{The Hermite Transform (HT)}

The Hermite transform (HT) [9] is a special case of polynomial transform, which is a technique of local decomposition of signals and can be regarded as an image description model. In this, the input image $L(x, y)$ is windowed with a local Gausian function $\omega(x-p, y-q)$ at the positions $(p, q)$ that conform the sampling lattice $S$. By replicating the window function over the sampling lattice, we can define the periodic weighting function as $W(x, y)=\sum_{(p, q) \in S} \omega(x-p, y-q)$. Then, the local information of each analysis window is expanded in terms of a family of orthogonal polynomials defined as

$$
G_{n-m, m}(x, y)=\frac{1}{\sqrt{2^{n}(n-m) ! m !}} H_{n-m}\left(\frac{x}{\sigma}\right) H_{m}\left(\frac{y}{\sigma}\right) .
$$

where $H_{i}\left(\frac{x}{\sigma}\right)$ denotes the ith Hermite polynomial orthogonal to the Gaussian window with standard deviation $\sigma$.

In every window function, the signal content is described as the weighted sum of polynomials $G_{m, n-m}(x, y)$ of $m$ degree in $x$ and $n-m$ in $y$. In a discrete implementation, the Gaussian window function may be approximated by the binomial window function and in this case, its orthogonal polynomials are known as Krawtchouck's polynomials.

In either case, the polynomial coefficients $L_{m, n-m}(p, q)$ are calculated convolving the original image $L(x, y)$ with the analysis filters $D_{m, n-m}(x, y)=$ $G_{m, n-m}(-x,-y) \omega^{2}(-x,-y)$, followed by subsampling at position $(p, q)$ of the sampling lattice $S$. That is, 


$$
L_{m, n-m}(p, q)=\int_{-\infty}^{+\infty} \int_{-\infty}^{+\infty} L(x, y) D_{m, n-m}(x-p, y-q) d x d y .
$$

The recovery process of the original image consists of interpolating the transform coefficients with the proper synthesis filters. This process is called inverse transformed polynomial and it is defined by

$$
\widehat{L}(x, y)=\sum_{n=0}^{\infty} \sum_{m=0}^{n} \sum_{(p, q) \in S} L_{m, n-m}(p, q) P_{m, n-m}(x-p, y-q) .
$$

where $P_{m, n-m}(x, y)=\left(G_{m, n-m}(x, y) \omega(x, y) / W(x, y)\right)$ are the synthesis filters of order $m$ and $n-m$ for $m=0, \ldots, n$ and $n=0, \ldots, \infty$.

\subsection{The Steered HT}

The Hermite transform has the advantage of high-energy compaction by adaptively steering the HT [12, 10. Steerable filters are a class of filters that are rotated copies of each filter, constructed as a linear combination of a set of basis filters. The steering property of the Hermite filters explains itself because this filters are products of polynomials with a radially symmetric window function. The $N+1$ Hermite filters of Nth-order form a steerable basis for each individual Nth-order filter. Because of this property, the Hermite filters at each position in the image adapt the local orientation content. In terms of orientation frequency functions, this property of the Hermite filters can be expressed by

$$
g_{m, n-m}\left(\theta-\theta_{0}\right)=\sum_{k=0}^{n} c_{m, k}^{n}\left(\theta_{0}\right) g_{n-k, k}(\theta) .
$$

where $c_{m, k}^{n}\left(\theta_{0}\right)$ is the steering coefficient. The orientation selectivity for the filter is expressed by

$$
g_{m, n-m}(\theta)=\sqrt{\left(\begin{array}{c}
n \\
m
\end{array}\right)} \cos ^{m} \theta \sin ^{n-m} \theta .
$$

For the directional Hermite decomposition, first, a HT was applied and then the coefficients were rotated toward the estimated local orientation $\theta$, according to a criterion of maximum oriented energy at each window position. This angle can be approximated as $\theta=L_{01} / L_{10}$, where $L_{01}$ and $L_{10}$ are a good estimated to optimal edge detectors in the horizontal and vertical directions respectively (Fig. 1] shows the HT and the steered HT over an image).

\subsection{The Multiresolution Directional-Oriented HT}

A multiresolution decomposition using the HT can be obtained through a pyramid scheme [6]. In a pyramidal decomposition, the image is decomposed into a 


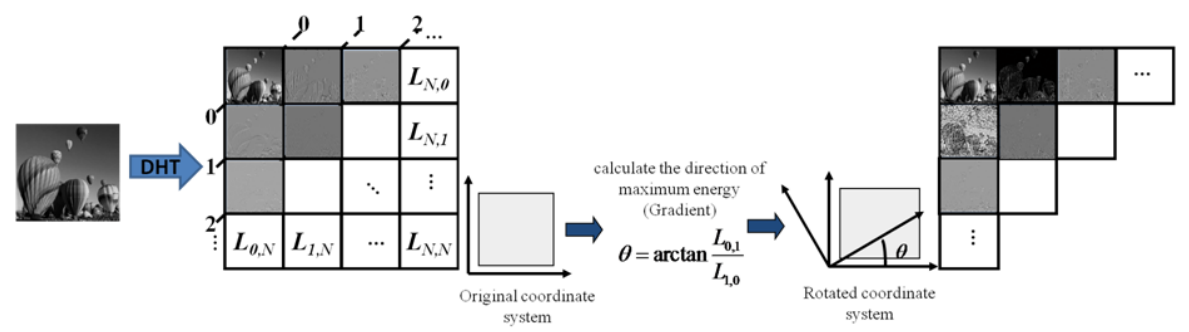

Fig. 1. The Discrete Hermite Transform (DHT) and the steered Hermite Transform over an image

number of band-pass or low-pass subimages, which are then subsampled in proportion to their spatial resolution. In each layer the zero order coefficients are transformed to obtain -in a lower layer- a scaled version of the one above. Once the coefficients of Hermite decomposition of each level are obtained, the coefficients can be projected to one dimension by its local orientation of maximum energy. In this way we obtain the multiresolution directional-oriented Hermite transform, which provides information about the location and orientation of the structure of the image at different scales.

\section{Proposed Image Fusion Algorithm}

Our approach aims at analyzing images by means of the HT, which allows us to identify perceptually relevant patterns to be included in the fusion process while discriminating spurious artifacts. As we have mentioned, the steered HT allows us to focus energy in a smaller number of coefficients, and thus the information contained in the first-order rotated coefficient may be sufficient to describe the edge information of the image in a particular spatial locality. If we extend this strategy to more than one level of resolution, then it is possible to obtain a better description of the image.

However, the success of any fusion scheme depends not only on the image analysis model but also on the fusion rule, therefore, instead of choosing for the usual selection operators based on the maximum pixel value, which often introduce noise and irrelevant details in the fused image, we seek a rule to consider the existence of a pattern in a region defined by a fixed-size window.

The general framework for the proposed algorithm includes the following stages. First a multiresolution HT of the input images is applied. Then, for each level of decomposition, the orientation of maximum energy is detected so that the coefficients can rotate, thus the first order rotated coefficient has the most information about edges. Afterwards, taking this rotated coefficient of each image we apply a linear dependence test. The result of this test is then used as a decision map to select the coefficients of the fused image in the multiresolution HT domain of the input images. If the original images are noisy, the decision map is applied on the multiresolution HT directional-oriented. The approximation coefficients in the case of HT are the zero order coefficients. In most multifocal and 


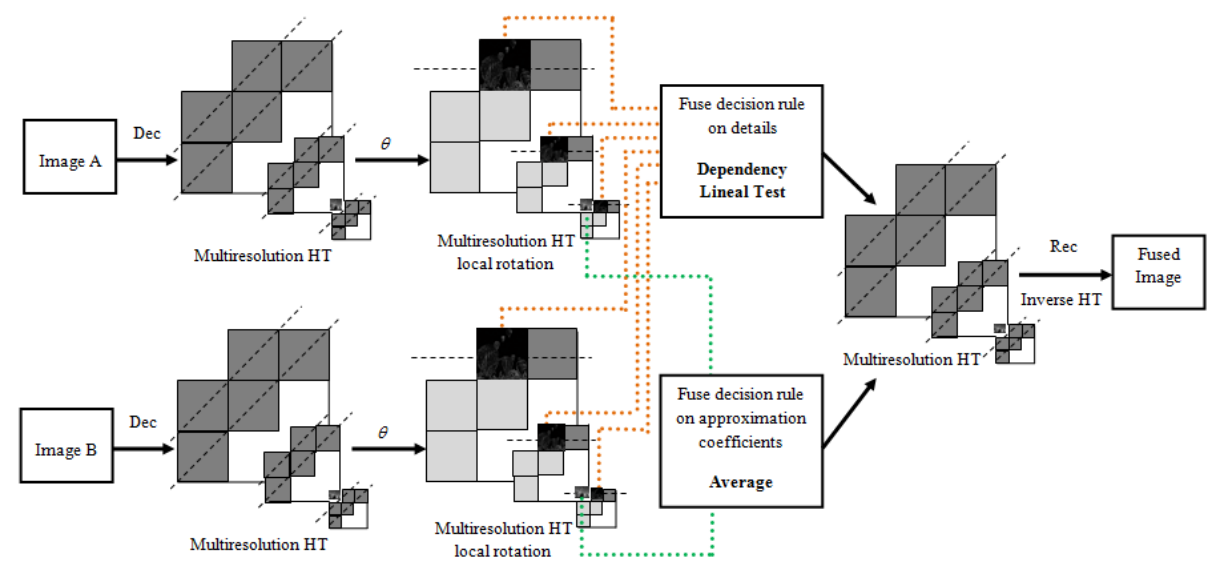

Fig. 2. Fusion scheme with the multiresolution directional-oriented Hermite Transform

multimodal applications the approximation coefficients of the input images are averaged to generate the zero order coefficient of the fused image, but this always depends on the application context. Finally, the fused image is obtained by applying the inverse multiresolution HT (Fig. 2] shows a simplified representation of this method).

\subsection{The Fusion Rule}

The linear dependence test evaluates the pixels inside a window of $w_{s} \times w_{s}$, if those pixels are linearly independent, then there is no relevant feature in the window. However, if the pixels are linearly dependent, it indicates the existence of a pattern. The fusion rule selects the coefficient with the highest dependency value. A higher value will represent a stronger pattern.

This approach has been reported in the literature in image fusion schemes that use the wavelet transform [1] and the curvelet transform [8; their basis is an empirical method proposed in [3], where the image is analyzed in small regions and each neighborhood of a pixel is expressed as a vector in which the linear dependence is calculated.

A simple and rigorous test for determining the linear dependence or independence of vectors is the Wronskian determinant, wich is defined for functions but can also be applied to vectors. The dependency of the window centered at a pixel $(i, j)$ is described in

$$
D_{A}(i, j)=\sum_{m=i-w_{s}}^{i+w_{s}} \sum_{n=j-w_{s}}^{j+w_{s}} L_{A}^{2}(m, n)-L_{A}(m, n) .
$$

where $L_{A}(m, n)$ is the first order steered Hermite coefficient of the source image $A$ with spatial position $(m, n)$. The coefficient of the fused HT is selected as the one with largest value of the dependency measure, then 


$$
L_{F}(i, j)=\left\{\begin{array}{ll}
L_{A}(i, j) & \text { if } D_{A}(i, j) \geq D_{B}(i, j) \\
L_{B}(i, j) & \text { if } D_{A}(i, j)<D_{B}(i, j)
\end{array} .\right.
$$

We apply this rule to all detail coefficients and average the zero order Hermite coefficients as

$$
L_{00_{F}}(i, j)=\frac{1}{2}\left[L_{00_{A}}(i, j)+L_{00_{B}}(i, j)\right] .
$$

\section{Experiments and Results}

The proposed algorithm was tested on several sets of multi-focus and multimodal images. Fig. 3 shows one of the multi-focus image sets used and the results of image fusion achieved with different fusion methods, all of them using the linear dependence test with a window size of $3 \times 3$, and two decomposition levels. By the HT, we used a Gaussian window with spread $\sigma=\sqrt{2}$, a subsampling factor $T=2$ between each pyramidal level. The DWT used was db4 and in the case of the $\mathrm{CW}$, the McClellan transform of 9-7 filters were used as directional filters and the wavelet db4 was used as pyramidal filters.

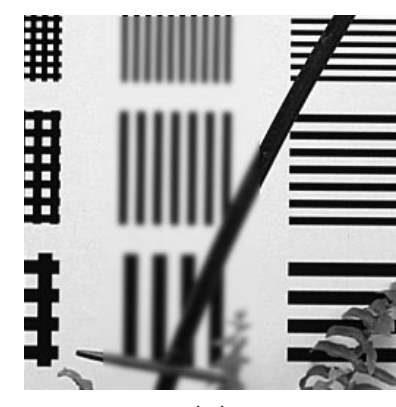

(a)

(d)

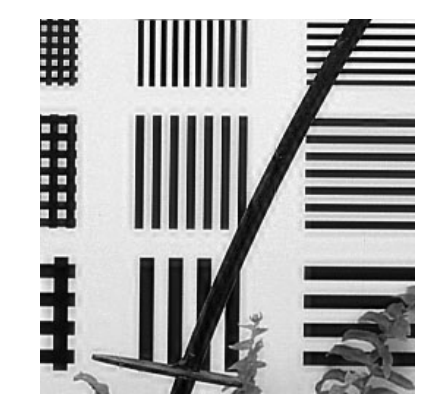

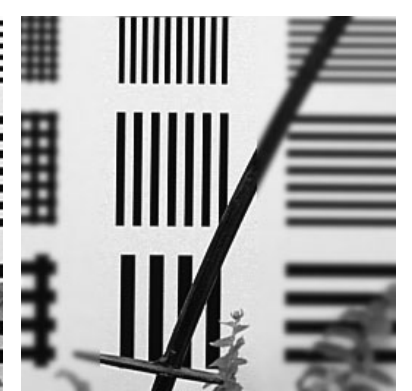

(b)

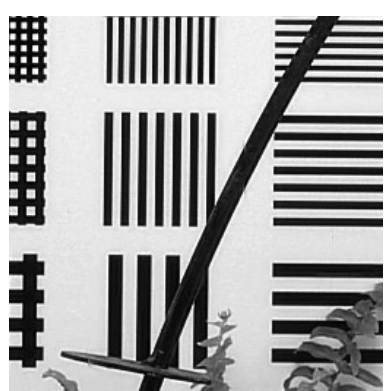

(c)

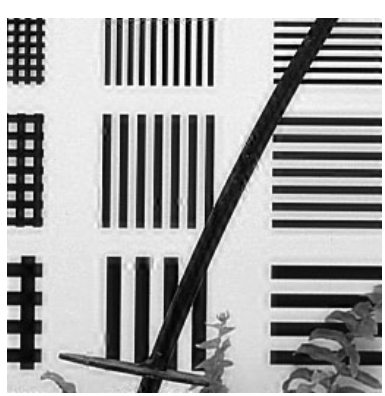

(e)

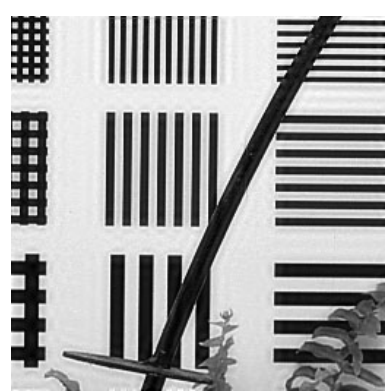

(f)

Fig. 3. Results of image fusion in multi-focus images, using different analysis techniques. (a) and (b) as the source images, c) HT, d) DWT, e) CW and f) CUW. 


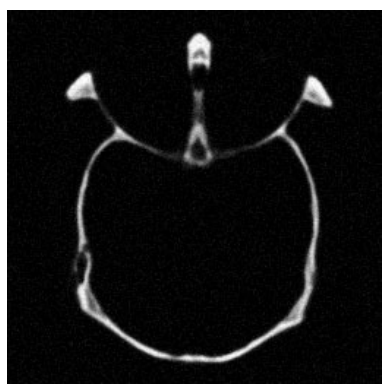

(a)

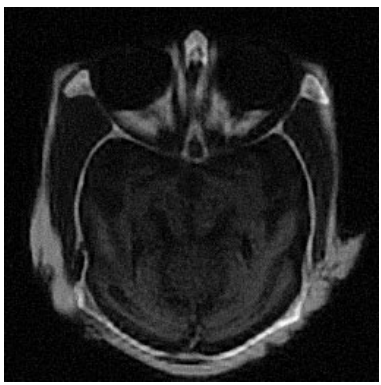

(d)

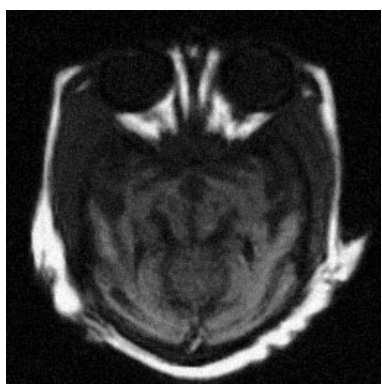

(b)

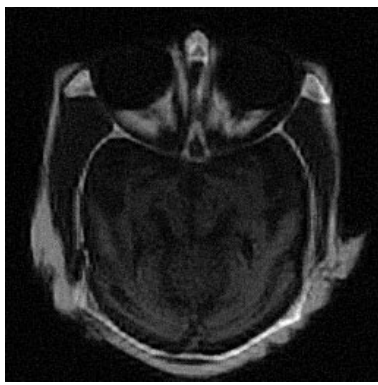

(e)

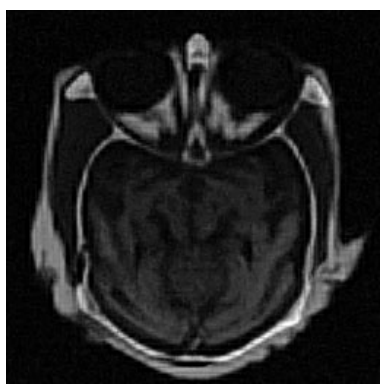

(c)

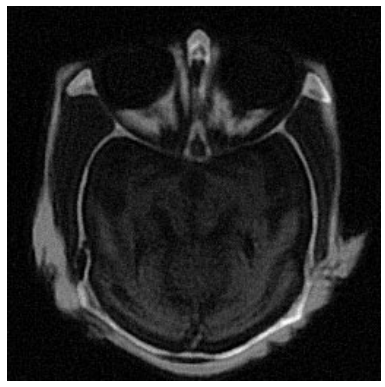

(f)

Fig. 4. Results of image fusion in noisy medical images, using different analysis techniques. (a) computed tomography (CT) and (b) magnetic resonance (MR) as the source images, c) HT, d) DWT, e) CW and f) CUW.

On the other hand, Fig. 4 shows the application in noisy medical images with the same described parameters. In this case, Gaussian noise with $\sigma=0.001$ was introduced to the original images.

From Figs. 3 and 4 , we can notice that the image fusion method based on the Hermite transform preserved the spatial resolution and information content of both images better. Moreover, our method shows a superior performance in noise reduction.

In order to quantitatively compare the proposed algorithm with the others, we evaluated our fusion results with several quality metrics: the peak signal to noise ratio (PSNR) defined in Eq. 9, the mean square error (MSE) defined in Eq. 10, the measure of structural similarity (SSIM) [13] defined in Eq. 11] and the Mutual information (MI) 14 defined in Eq. 12

$$
\begin{gathered}
P S N R=10 \log _{10} \frac{255^{2}(M N)}{\sum_{i=1}^{M} \sum_{j=1}^{N}[F(i, j)-R(i, j)]^{2}} . \\
M S E=\frac{\sum_{i=1}^{M} \sum_{j=1}^{N}[F(i, j)-R(i, j)]^{2}}{M N} .
\end{gathered}
$$


where $F(i, j)$ denotes the intensity of the pixel of the fused image and $R(i, j)$ denotes the intensity of the pixel of the original image.

$$
\operatorname{SSIM}(R, F)=\frac{\sigma_{R F}}{\sigma_{R} \sigma_{F}} \frac{2 \mu_{R} \mu_{F}}{\left(\mu_{R}\right)^{2}+\left(\mu_{F}\right)^{2}} \frac{2 \sigma_{R} \sigma_{F}}{\sigma_{R}^{2} \sigma_{F}^{2}} .
$$

where $\mu_{R}$ is the original image mean and $\mu_{F}$ the fused image mean; $\sigma$ is the variance and $\sigma_{R F}$ is the covariance.

$$
M I_{F}^{A B}=M I_{F A}(F, A)+M I_{F B}(F, B) .
$$

where $M I_{F A}(F, A)=\sum P_{F A}(F, A) \log \left[\left(P_{F A}(F, A)\right) /\left(P_{F}(F) P_{A}(A)\right)\right]$ is the amount of information that belongs to image $A$ contained in the fused image, where $P_{F}$ and $P_{A}$ are the marginal probability densisty functions of images $F$ and $A$ respectively, and $P_{F A}$ is the joint probability density funtion of both images.

Tab. 1 shows the performance of the method using different image analysis techniques with the same fusion rule. The values are the average of the tests performed on multifocal and medical images. Altogether we used 11 sets of images, in 6 of which, we compared with a ground truth. These ground truths were obtained from synthetic images.

Table 1. Performance measurement applying the fusion rule based on linear dependency with different methods

\begin{tabular}{lrrrrr}
\hline Fusion method & $M S E$ & $P S N R$ & $S S I M$ & $M I_{F}^{A B}$ \\
\hline Hermite Transform & 127.055 & 36.425 & 0.9640 & 6.130 \\
Wavelet Transform & 148.889 & 34.627 & 0.9574 & 5.595 \\
Contourlet Transform & 177.466 & 30.836 & 0.9477 & 5.535 \\
Curvelet Transform & 164.296 & 31.608 & 0.9496 & 5.609 \\
\hline
\end{tabular}

\section{Conclusions}

We have presented a multiresolution image fusion method based on the directional-oriented HT, which uses a linear dependency test as fusion rule. We have experimented with this method for multi-focus and multi-modal images and we have obtained good results, even in the presence of noise. Both subjective and objective results show that the proposed scheme outperforms other existing methods.

The HT has proved to be an efficient model for the representation of images because derivatives of Gaussian are the basis functions of this transform, which optimally detect, represent and reconstruct perceptually relevant image patterns, such as edges and lines.

Acknowledgments. This work was supported by UNAM grants IN113611 and IX100610. 


\section{References}

1. Aguilar-Ponce, R., Tecpanecatl-Xihuitl, J.L., Kumar, A., Bayoumi, M.: Pixel-level image fusion scheme based on linear algebra. In: IEEE International Symposium on Circuits and Systems ISCAS 2007, New Orleans, pp. 2658-2661 (2007)

2. Contourlet toolbox, http://www. mathworks.com/matlabcentral/fileexchange/8837

3. Durucan, E., Ebrahimi, T.: Change detection and background extraction by linear algebra. Proceedings of the IEEE 89(10), 1368-1381 (2001)

4. Escalante-Ramírez, B.: The Hermite transform as an efficient model for local image analysis: An application to medical image fusion. Comput. Electr. Eng. 34(2), 99-110 (2008)

5. Escalante-Ramírez, B., López-Caloca, A.: The Hermite transform: an efficient tool for noise reduction and image fusion in remote sensing. In: book: Image Processing for Remote Sensing, pp. 539-557. CRC Press, Boca Raton (2006)

6. Escalante-Ramírez, B., Silván-Cárdenas, J.L.: Advanced modeling of visual information processing: A multi-resolution directional-oriented image transform based on Gaussian derivatives. Signal Processing: Image Communication 20(9-10), 801-812 (2005)

7. Hill, P., Canagarajah, N., Bull, D.: Image Fusion using Complex Wavelets. In: Proc. 13th British Machine Vision Conference, pp. 487-496 (2002)

8. Mahyari, A., Yazdi, M.: A novel image fusion method using curvelet transform based on linear dependency test. In: International Conference on Digital Image Processing, pp. 351-354 (2009)

9. Martens, J.-B.: The Hermite transform-theory. IEEE Transactions on Acoustics, Speech and Signal Processing 38(9), 1595-1606 (1990)

10. Martens, J.-B.: Local orientation analysis in images by means of the Hermite transform. IEEE Transactions on Image Processing 6(8), 1103-1116 (1997)

11. The Curvelet.org team, http://www.curvelet.org/software.html

12. Van Dijk, A., Martens, J.-B.: Image representation and compression with steered Hermite transforms. Signal Processing 56(1), 1-16 (1997)

13. Wang, Z., Bovik, A., Sheikh, H., Simoncelli, E.: Image quality assessment: from error visibility to structural similarity. IEEE Transactions on Image Processing 13, 600-612 (2004)

14. Wang, Q., Yu, D., Shen, Y.: An overview of image fusion metrics. In: Conference on Instrumentation and Measurement Technology, pp. 918-923 (2009)

15. Yang, L., Guo, B.L., Ni, W.: Multimodality medical image fusion based on multiscale geometric analysis of contourlet transform. Neurocomputing 72(1-3), 203-211 (2008)

16. Young, R.: The Gaussian derivative theory of spatial vision: analysis of cortical cell receptive field line-weighting profiles. Integration. Technical report, General Motors Research (1986) 